\title{
The Effects of Different Exercise Intensities and Modalities on Cortisol Production in Healthy Individuals: A Review
}

Short Review

\begin{abstract}
Ricardo Torres ${ }^{1}$, Panagiotis Koutakis ${ }^{2}$, and Jeffrey S. Forsse ${ }^{1^{*}}$

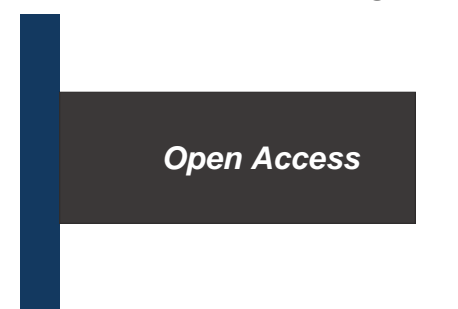

${ }^{1}$ Department of Health Human Performance and Recreation; Baylor University, Waco, TX, USA

${ }^{2}$ Clinical Muscle Biology Lab, Department of Biology, Baylor University, Waco, TX, US A

Published: October 28, 2021

Abstract

Cortisol is a hormone that is secreted in response to physiological stress. Exercise contributes significantly to changes in circulating cortisol concentrations. With exercise, there is increased activation of the sympathetic system to stimulate the release of adrenocorticotropic hormone, which releases cortisol into the blood. Current research has predominately studied the effects that aerobic exercise and resistance training have on cortisol production. Prolonged aerobic exercise, especially at higher intensities, significantly elevates cortisol concentrations when compared to similar duration and intensities of resistance exercise. Age, gender, physical fitness level, exercise intensity, training status, and modality are all variables that influence the production of cortisol. Elevated cortisol concentrations are highly indicative of muscle catabolism, increasing the loss of lean muscle tissue. This is a significant health concern for the growing elderly population. The rate of cortisol production changes as an individual ages and has been observed to have differing responses to exercise intensities in males and females. Cortisol production is correlated with exercise intensity and duration but does not increase the same across all exercise intensities. Higher exercise intensities and duration appear to be the main contributing factors that influence the production of cortisol, increasing the potential for muscle catabolism and muscle loss.

ISSN: 2640-2572
\end{abstract}

Key Words: Aerobic Exercise, Resistance Training,

Corresponding author: Jeffrey S. Forsse

Introduction

Circulating cortisol concentrations are always present in the blood and undergo fluctuations in concentrations due to physiologic and psychologic stressors ${ }^{1}$. The release of epinephrine and norepinephrine by the activation of the sympathetic nervous system stimulates the hypothalamus to release corticotropin-releasing hormone $(\mathrm{CRH})$ from the anterior pituitary gland ${ }^{2}$. CRH stimulates the pituitary synthesis of adrenocorticotropic hormone (ACTH) via the hypothalamic-pituitary-adrenal (HPA) axis. ACTH acts on the adrenal cortex to release cortisol ${ }^{3}$. Cortisol levels are 
regulated through negative feedback of reduced ACTH ${ }^{1}$. Increases in ACTH signal the hypothalamus to lower the production of the $\mathrm{CRH}$. Changes in exercise intensity, duration, training status, and mode of exercise elicits changes in circulating blood cortisol concentrations. Cortisol is taken up by the liver, skeletal muscle, and adipose tissue to activate multiple energy pathways. In the adipose tissue, cortisol promotes the hydrolysis of triglycerides to produce glycerol and free fatty acids ${ }^{4}$. In the skeletal muscle tissue, cortisol facilitates the initiation of protein degradation, converting proteins to amino acids. Elevated cortisol concentrations promote the degradation of skeletal muscle by increasing muscle catabolism, along with gluconeogenesis. This produces a greater rate of muscle catabolism compared to anabolism, which decreases lean muscle mass ${ }^{5}$. In the liver, elevated cortisol concentrations may cause the liver to begin gluconeogenesis, generating a surplus in energy production from carbohydrates. Though higher cortisol concentrations stimulate adverse physiological effects, cortisol is also important in maintaining normal physiological processes (e.g., immune and stress response, and glucose and protein homeostasis) ${ }^{6}$. Exercise intensities influence essential hormonal responses during exercise, specifically in cortisol 7 . A common comparison is between high-intensity and low-intensity exercise. Training modalities are increasingly migrating to encompass more high-intensity interval exercises (HIIE), which include aerobic training (AT) and resistance training (RT) ${ }^{8-10}$. These training modalities and intensities affect cortisol production both positively and negatively ${ }^{10-12}$. There are additional variables to consider, such as age, gender, time of day, and physical activity levels that influence cortisol responses to exercise intensities ${ }^{13-}$ 15. Higher exercise intensities are positively correlated to elevated cortisol concentrations; however, there are exceptions to this general claim ${ }^{1}$. Cortisol was shown to be significantly lower in 90\% 1-RM when compared to $75 \% 1$-RM in resistance-trained individuals ${ }^{10}$. The same was observed in eight trained men participating in high and low volume rowing protocol where cortisol was not significantly changed in resting values ${ }^{16}$. This potentially could be attributed to participants' level of training and conditioning. However, numerous studies have shown increased cortisol concentrations when correlated with intensity levels in both trained and untrained individuals. Low versus moderateintensity exercises decreased resting cortisol concentrations after untrained men completed an eight-week RT program 13. When evaluating intensity levels, time has been shown to contribute to increases and decreases in cortisol production significantly ${ }^{17,18}$. In addition, the hormonal effect that cortisol has on muscle function is dynamic to maintaining health and performance for young and older individuals. Age has been shown to significantly impact the production of cortisol and exacerbates the rate of muscle atrophy and sarcopenia ${ }^{14,15}$. Aside from these variables that affect the hormonal responses of cortisol production, gender has a significant impact on cortisol and is a contributing factor to health and performance ${ }^{19}$. Therefore, the purpose of this review was to examine the impact that different exercise intensities and modalities have on cortisol production in healthy men and women.

\section{Literature Review Methods}

A literature review was performed to examine the effects of aerobic and resistance exercise intensities on cortisol production. The search was performed on PubMed, Medline, and EBSCO Host. The search terms used were "resistance training cortisol," "aerobic exercise cortisol," "anaerobic exercise cortisol," "combined exercise cortisol," "exercise cortisol men," "exercise cortisol women," and "resistance training cortisol men/women." The search was concluded on September 1, 2021. Criteria required for research articles to be included in the review. Cortisol had to be measured during multiple time points with a minimum of pre and post-exercise intervention (aerobic, resistance, or both). Males and females, all ages, serum, plasma, and salivary measures of cortisol that were collected through a blood draw or swabs collected orally, including all exercise intensities, and healthy individuals at different stages of development. The literature review produced nineteen articles focusing on aerobic exercise, thirteen articles focusing on resistance training, and four articles focused on combined aerobic exercise and resistance training's influence on cortisol production. A single investigator performed the methodological quality of included studies.

\section{Aerobic Exercise Intensities Impact on Cortisol Production}

With aerobic exercise, cortisol has been observed to increase depending on intensity levels ${ }^{20-23}$. Exercise modalities, intensity, training status, and outcomes in cortisol production in males and females are displayed in Table 1. In one study, seven endurance-trained athletes completed three exercise protocols. Exercise conditions included HIIE, steadystate exercise (SSE), and prolonged endurance exercise ${ }^{24}$. SSE displayed no significant changes in cortisol concentrations, differing from HIIE, which displayed slight increases in cortisol production. However, prolonged endurance exercise exhibited the greatest changes in cortisol, increasing circulating levels by two-fold ${ }^{25}$. Time appears to be the main contributing factor that influences cortisol concentrations with aerobic exercise ${ }^{20-29}$. In a study involving eight trained male athletes, cortisol concentrations were significantly elevated after completing a 120-minute acute bout of sub-maximal exercise. When comparing 120 to 45 -minute time trials, cortisol had a greater increase with the longer exercise protocol ${ }^{30}$. However, when relating HIIE to SSE for the same amount of time, there is a greater cortisol response to HIIE than SSE. It was concluded that cortisol responded less to continuous exercise in higher 
trained individuals ${ }^{31}$. When an acute bout of HIIE was divided up into very short bouts (4x30s sprints) and compared to high volume endurance (HVE) training $\left(50 \% \mathrm{VO}_{2 \max }\right.$ for $\left.60 \mathrm{~min}\right)$. HIIE elicited significant increases in cortisol with no significant change in the HVE group ${ }^{3}$. During an incremental cycle ergometer test, an intensity of $60 \% \mathrm{VO}_{2 \text { peak }}$ stimulated a significant change in cortisol levels compared to baseline. Cortisol concentrations were further elevated following the exercise protocol ${ }^{18}$. Additionally, Hill et al. ${ }^{1}$ had moderately trained men participate in an acute treadmill test set at $40 \%, 60 \%, 80 \%$, and $100 \% \mathrm{VO}_{2 \max }$. After comparing moderate-intensity exercise to the low-intensity (40\%) exercise session, there was a significant increase in cortisol concentrations in an exercise session consisting of $60-80 \%$. These changes in cortisol concentrations are more than likely due to greater sympathetic activation by exercise intensities greater than 50-60\%. However, cortisol and ACTH levels in low-intensity exercise may also be dependent on circulating glucose concentrations during prolonged exercise ${ }^{32}$. Training status poses a significant impact on cortisol responses during aerobic exercise. There appears to be a blunting or reduced sensitivity when participating in higherintensity exercises and SEE 16,20,33,34. An acute low-to-moderate-intensity exercise of $60 \%$ for 50 minutes once a week for 12 weeks in sedentary young males was shown to have no significant changes in cortisol concentrations at lower exercise intensities. Exhibiting that a sedentary lifestyle could have adverse effects on metabolic hormones ${ }^{16}$. In endurance-trained males, SEE attenuated cortisol production compared to HIIE and prolonged endurance exercisedemonstrating that training adaptations contributed heavily to attenuating cortisol production in different HIIE and SEE modalities ${ }^{17}$.

\section{Resistance Training Intensities Impact on Cortisol Production}

Cortisol production is significantly correlated to RT modalities and intensities. Specific effects of RT on cortisol concentrations are displayed in Table 2. In resistance-trained individuals, cortisol was observed to be affected differently in three acute protocols consisting of hypertrophy (75\% 1-RM squats), strength ( $90 \% 1$-RM squats), and power ( $0 \%$ 1-RM box jumps). Cortisol was assessed over 48 hours with significant changes presenting immediately post-exercise (IPE). Strength and power groups were decreased while the hypertrophy group was significantly elevated ${ }^{10}$. Similarly, Szivak et al. ${ }^{9}$ had nine males and nine females who were RT trained to participate in acute RT, consisting of high intervals at 75\% 1-RM. Cortisol concentrations increased at IPE, 15, and 60 minutes, peaking IPE in both males and females. Strength training appears to display diminished acute changes in cortisol concentrations when heavier RT protocols are combined with lower reps and longer rest periods between sets. Higher RT intensities (>75\%) produced greater cortisol responses when compared to low- and moderate-intensity levels ${ }^{35-37}$. High repetitions with short rest periods increase cortisol levels in RT primarily due to greater sympathetic activation that is correlated with increases in heart rate. When high-intensity RT (75\% 1-RM for 15 reps) was compared to moderate-intensity RT ( $60 \%$ 15 reps), the results demonstrated that high-intensity elicits a significant ( $68 \%$ increase) cortisol response within the first 3 hours post-exercise, while moderate-intensity had no significant changes. Each exercise condition implemented 2 minute rest periods in-between each set, totaling 90 minutes of exercise time ${ }^{38}$. Training status in RT is significantly correlated to cortisol production in varied resistance exercise intensities. When comparing untrained men to moderately or highly trained men, there are different responses to cortisol production. Arazi et al. ${ }^{13}$ observed significant decreases in resting cortisol levels after completing $3 \times 8$ weeks of moderate-intensity RT exercises of squats and bench press in untrained men ${ }^{13}$. Acute RT produces different cortisol responses in untrained individuals that differ from regular exercise. Physically fit, untrained individuals who participate in an acute bout of RT responded differently than trained individuals. Nine physically fit untrained males completed high-intensity normal movement (HINM) (80\%), low-intensity slow movement (LISM) (40\%), and low-intensity normal movement (LINM) (40\%). HINM increased blood cortisol IPE and returned to baseline after 30 minutes. LISM significantly increased cortisol levels IPE and levels remained elevated for 30 minutes. LINM gradually decreased circulating cortisol from preexercise until 30 minutes' post-exercise, returning to baseline post 30 minutes ${ }^{39}$. Further supporting exercise intensity and time having the greatest influence on sympathetic activation.

\section{Combined Resistance Training and Aerobic Exercise Intensities Impact on Cortisol Production}

While done separately, aerobic exercise and RT affect cortisol production due to the exercise intensity and duration. Multi-facet exercises that incorporate both aerobic exercise and RT produce varied responses. Shrek et al. ${ }^{40}$ observed ten male rock climbers who performed 55' submaximal vertical climbs for thirty minutes or to exhaustion, whichever came first. After comparing the hormonal responses, pre-exercise, and IPE, cortisol did not significantly increase due to the large variability in cortisol concentrations. Three subjects showed a decrease, two subjects showed a minimal response, and five subjects showed an increase. ${ }^{40}$ While the protocol, according to Shrek et al. ${ }^{40}$ was more aerobic than expected, and rock climbing was a more isometric and concentric contraction, this is potentially the reason for such varied results. In studies that implemented acute bouts of $\mathrm{HIIE}$ aerobic exercise of $75 \%$ of $\mathrm{VO}_{2 \max }$ elicited higher cortisol responses when compared to fast tempo eccentric contractions. ${ }^{34,37}$ 
Table 1. Production of Cortisol in Response to Different Aerobic Exercise Intensities

\begin{tabular}{|c|c|c|c|c|c|c|c|c|}
\hline References & $\begin{array}{l}\text { Mode of } \\
\text { Exercise }\end{array}$ & Subjects & $\begin{array}{l}\text { Training } \\
\text { Status }\end{array}$ & Intensity Level & $\begin{array}{l}\text { Intervention } \\
\text { Length }\end{array}$ & $\begin{array}{c}\text { Biological } \\
\text { Tissue }\end{array}$ & $\begin{array}{l}\text { Cortisol } \\
\text { Collection } \\
\text { Times }\end{array}$ & Cortisol Outcomes \\
\hline Rojas Vega et al (2006) & CE & $\begin{array}{l}\mathrm{n}=8 \\
\sigma^{\lambda}=8\end{array}$ & $\begin{array}{l}\text { Trained } \\
\text { Athletes }\end{array}$ & HIIE (Incremental) & Acute & Serum & $\begin{array}{l}\text { Pre, } 10 \text {-w-up, IPE, } \\
3,6,10, \& 15 \mathrm{~min}\end{array}$ & $\begin{array}{l}\text { HIIE = Sig. } \uparrow @ 10 \& 15 \\
\text { mins }\end{array}$ \\
\hline Hew-Butler et al (2008) & A & $\begin{array}{l}\mathrm{n}=7 \\
\delta=7\end{array}$ & $\begin{array}{l}\text { Trained } \\
\text { Endurance } \\
\text { Athletes }\end{array}$ & $\begin{array}{l}\text { HIIE - Exhaustion } \\
\text { SSE - } 60 \mathrm{~min} \\
\text { PEE - } 56 \mathrm{KM}\end{array}$ & Acute & Serum & Pre-and Post & $\begin{array}{l}\text { HIIE }-\uparrow \text { Slightly } \\
\text { SEE }-\downarrow \text { (No sig. changes) } \\
\text { PEE }-\uparrow 2 \mathrm{X}\end{array}$ \\
\hline Hill et al (2008) & A & $\begin{array}{l}\mathrm{n}=12 \\
\delta=12\end{array}$ & $\begin{array}{l}\text { Moderately } \\
\text { Trained }\end{array}$ & $\begin{array}{l}40 \%, 60 \%, 80 \% \\
\mathrm{VO}^{2} \max \end{array}$ & Acute & Serum & $\begin{array}{l}\text { Pre \& Post for } \\
\text { each exercise } \\
\text { condition }\end{array}$ & $\begin{array}{l}60 \% \text { and } 80 \% \text { was sig. } \uparrow \\
\text { compared to } 40 \%\end{array}$ \\
\hline Witard et al (2012) & CE & $\begin{array}{l}\mathrm{n}=8 \\
\delta=8\end{array}$ & Trained & $\begin{array}{l}120 \text { min sub-max } \\
\text { test followed by } 45 \\
\text { min time trial. }\end{array}$ & $\begin{array}{l}1 \text { week moderate } \\
\text { and } 1 \text {-week high } \\
\text { intensity }\end{array}$ & Serum & $\begin{array}{l}\text { Pre, during sub- } \\
\text { max, during time } \\
\text { trial, } 1 \text { hour PE }\end{array}$ & $\begin{array}{l}\text { PE C levels in moderate } \\
\text { intensity was } \uparrow \text { greater than } \\
\text { high intensity }\end{array}$ \\
\hline Deuster et al (1989) & A & $\begin{array}{l}\mathrm{n}=21 \\
\hat{O}=7 \text { untrained, } \\
7 \text { moderately, } 7 \\
\text { highly }\end{array}$ & $\begin{array}{l}\text { Untrained, } \\
\text { moderately } \\
(\mathrm{M}) \text {, and } \\
\text { highly }(\mathrm{H})\end{array}$ & $50 \%, 70 \%$ and $90 \%$ & $\begin{array}{l}1 \text { x week x } 3 \\
\text { weeks }\end{array}$ & Serum & $\begin{array}{l}15 \text { pre, pre, during } \\
\text { exercise }(15 \& 25) \text {, } \\
\text { PE, } 35,45,55,65 \text {, } \\
85,105 \text {, and } 125\end{array}$ & $\begin{array}{l}\mathrm{C} \uparrow \text { greater in exercise } \\
\text { intensities of } 50 \%-90 \% \text {, and } \\
\text { was greater in untrained men } \\
\text { compared to } \mathrm{M} / \mathrm{H} \text { trained }\end{array}$ \\
\hline $\begin{array}{l}\text { WJ Kraemer et al } \\
(1995)\end{array}$ & $\mathrm{RT} / \mathrm{A}$ & $\begin{array}{l}\mathrm{n}=35 \\
\hat{\sigma}=35\end{array}$ & $\begin{array}{l}\text { Health } \\
\text { Physically } \\
\text { Fit }\end{array}$ & $\begin{array}{l}\mathrm{HI} / \mathrm{SE}, \mathrm{HI} / \mathrm{S}, \\
\mathrm{HI} / \mathrm{E}, \text { Upper Body } \\
\text { HI/SE, Control }\end{array}$ & 2x12 weeks; & Serum & Pre, $4,8,12$ weeks & $\begin{array}{l}\mathrm{HI} / \mathrm{SE}-\uparrow \text { pre- } 12 \text { weeks; } \\
\mathrm{HI} / \mathrm{S}-\text { initially } \uparrow \text { then } \downarrow \text { by } \\
\text { week } 12 ; \mathrm{HI} / \mathrm{E}-\uparrow \text { pre- } 12 \\
\text { weeks; Upper Body HI/SE - } \\
\text { initially } \uparrow \text { then } \downarrow \text { by week } 12\end{array}$ \\
\hline
\end{tabular}


2021, Volume 4 (Issue 4): 19

\begin{tabular}{|c|c|c|c|c|c|c|c|c|}
\hline Wahl et al (2010) & $\mathrm{CE}$ & $\begin{array}{l}n=11 \\
\widehat{o}=11\end{array}$ & $\begin{array}{l}\text { Health } \\
\text { Physically } \\
\text { Fit }\end{array}$ & $\begin{array}{l}\text { HIIE }(2 \times 4(30 \text { s)) } \\
\text { vs High Volume } \\
\text { Endurance Training } \\
\text { (HVT } 1 \text { hour) }\end{array}$ & Acute & Serum & $\begin{array}{l}\text { Pre, } 10-, 60-, \& \\
\text { 240-mins PE }\end{array}$ & $\begin{array}{l}\text { C sig. } \uparrow \text { in HIIE @ } 10 \text { mins } \\
\text { PE; but did not have any sig. } \\
\text { during HVT }\end{array}$ \\
\hline Maresh et al (2006) & A & $\begin{array}{l}n=9 \\
\hat{o}=9\end{array}$ & $\begin{array}{l}\text { Trained } \\
\text { Endurance } \\
\text { Athletes }\end{array}$ & $\begin{array}{l}70 \% \text { and 85\%@ } \\
\text { Hydration } \\
\text { (Euhydrated/Hypo } \\
\text { hydrated 5\% body } \\
\text { mass) }\end{array}$ & Acute & Serum & $\begin{array}{l}\text { Pre, IPE, \& } 20 \\
\text { mins }\end{array}$ & $\begin{array}{l}\mathrm{C} \text { was } \uparrow \text { in both exercise } \\
\text { intensities in hypo hydration } \\
\text { vs euhydration@ @ pre, } 20 \text { min } \\
\text { PE }\end{array}$ \\
\hline Viru et al (2008) & $\mathrm{CE}$ & $\begin{array}{l}n=8 \\
\delta=8\end{array}$ & $\begin{array}{l}\text { Health } \\
\text { Physically } \\
\text { Fit }\end{array}$ & $\begin{array}{l}\text { Low intensity } 60 \% \\
(3 \text { min incremental } \\
\text { WL) }\end{array}$ & Acute & Serum & Pre \& each stage & $\begin{array}{l}\text { Sig. } \uparrow \text { after } 1 \text { st stage \& } \\
\text { remained stable until last } 2 \\
\text { stages then } \uparrow \text { more. }\end{array}$ \\
\hline $\begin{array}{l}\text { Buono and Yeager } \\
\text { et al (1991) }\end{array}$ & $\mathrm{CE}$ & $\begin{array}{l}n=7 \\
\delta=7\end{array}$ & $\begin{array}{l}\text { Health } \\
\text { Physically } \\
\text { Fit }\end{array}$ & $\begin{array}{l}\mathrm{VO}_{2 \max }(\mathrm{WL} \text { was } \\
\text { increased by } 50 \\
\text { watts each stage) }\end{array}$ & Acute & Serum & $\begin{array}{l}\text { Pre, Each stage, } \\
\text { PE }\end{array}$ & $\begin{array}{l}\mathrm{C} \text { was only sig. } \uparrow \text { at } \\
\text { exhaustion }\end{array}$ \\
\hline Tremblay et al (2005) & A & $\begin{array}{l}n=8 \\
\hat{o}=8\end{array}$ & $\begin{array}{l}\text { Trained } \\
\text { Endurance } \\
\text { Athletes }\end{array}$ & $\begin{array}{l}\text { Treadmill - 40, 80, } \\
120 \text { mins @ 55\% }\end{array}$ & Acute & Serum & $\begin{array}{l}\text { Pre, } 1,2,3, \& 4 \\
\text { hours from start of } \\
\text { run }\end{array}$ & $\begin{array}{l}C \text { only } \uparrow \text { at } 120 \text { mins, all } \\
\text { other protocols } \downarrow \text { across time. }\end{array}$ \\
\hline Näveri et al (1985) & $\mathrm{CE}$ & $\begin{array}{l}n=8 \\
\hat{o}=8\end{array}$ & Trained & $\begin{array}{l}10 \min =63 \% ; 10 \\
\min =83 \% ; 5-7 \min \\
=\mathrm{VO}^{2} \max \end{array}$ & Acute & Serum & Pre-and Post & Only sig. $\uparrow$ was in $\mathrm{VO}^{2}$ max \\
\hline Peake et al (2004) & $\mathrm{CE}$ & $\begin{array}{l}\mathrm{n}=10 \\
\hat{\delta}=10\end{array}$ & $\begin{array}{l}\text { Trained } \\
\text { Endurance } \\
\text { Athletes }\end{array}$ & $\begin{array}{l}\mathrm{HIE}-\mathrm{VO}_{2 \max }, \mathrm{MIE} \\
\text { - continuous @ } 80 \%\end{array}$ & Acute & Serum & $\begin{array}{l}\text { Pre, IPE, 1- \& 2- } \\
\text { hour PE }\end{array}$ & $\begin{array}{l}\mathrm{C} \uparrow \text { IPE in HIE; MIE no sig. } \\
\text { changes }\end{array}$ \\
\hline Buono et al (1986) & $\mathrm{CE}$ & $\begin{array}{l}n=6 \\
\lambda=6\end{array}$ & * & $\begin{array}{l}120 \% \text { of } \mathrm{VO}_{2 \max } \text { for } \\
1 \mathrm{~min}\end{array}$ & Acute & Serum & $\begin{array}{l}\text { Pre, IPE, 5-, 15-, } \\
\& 30 \text {-mins PE }\end{array}$ & C sig. $\uparrow @ 15$ mins PE \\
\hline Farrell et al (1983) & A & $\begin{array}{l}\mathrm{n}=6 \\
\hat{\lambda}=3 \\
+=3\end{array}$ & $\begin{array}{l}\text { Health } \\
\text { Physically } \\
\text { Fit }\end{array}$ & $\begin{array}{l}\text { Treadmill runs @ } \\
65 \% \text { and } 80 \% \text { for } 20 \\
\text { mins. Incremental } \\
\text { run till exhaustion }\end{array}$ & Acute & Serum & Pre-and IPE & $\begin{array}{l}\mathrm{C} \uparrow \text { similarly across all } \\
\text { exercise conditions }\end{array}$ \\
\hline $\begin{array}{l}\text { Kuoppasalmi et al } \\
(1980)\end{array}$ & A & $\begin{array}{l}\mathrm{n}=5(\mathrm{YM}) \\
\hat{O}=5\end{array}$ & $\begin{array}{l}\text { Trained } \\
\text { Sprinters } \\
\text { Athletes }\end{array}$ & $\begin{array}{l}\text { MI - } 90 \text { min run @ } \\
4.3 \\
\min / \mathrm{km} ; \mathrm{HI}-45 \\
\min \text { run @ } 3.3 \\
\min / \mathrm{km}\end{array}$ & Acute & Serum & Pre-and IPE & $\mathrm{HI} \uparrow \mathrm{C} 27 \%$ and $\mathrm{MI} \uparrow \mathrm{C} 43 \%$ \\
\hline Meckel et al (2009) & A & $\begin{array}{l}n=6 \\
\hat{O}=6\end{array}$ & Trained & $\begin{array}{l}4 \times 250 \mathrm{M} \times 3 \mathrm{~min} \\
\text { rest in between @ } \\
80 \% \text { intensity }\end{array}$ & Acute & Serum & $\begin{array}{l}\text { Pre, IPE each run, } \\
1 \text { hour PE }\end{array}$ & $\begin{array}{l}\text { C did not sig. change during } \\
\text { the protocol }\end{array}$ \\
\hline
\end{tabular}

Abbreviations: A = Aerobic; B = Baseline; BF = Blood Flow; CE = Cycle Ergometer; C = Cortisol; E = Endurance; EM = Elderly Men; EW = Elderly Women; HIIE = High Intensity Interval Exercise; MM = Middle-age Men; MW = Middle-age Women; PEE = Prolonged Endurance Exercise; H = Hypertrophy; IPE = Immediately Post-Exercise; P = Power; PE = Post Exercise; R $=$ Running; RT = Resistance Training; $\mathrm{S}=$ Strength; SSE = Steady State Exercise; WL = Workload; YM = Young Men; YW = Young Women; $\downarrow=$ Decreased; $\uparrow=$ Increased; $\widehat{O}=\mathrm{Male} ;$ Female; $*=$ Unknown 
Table 2. Production of Cortisol in Response to Different Resistance Training Intensities

\begin{tabular}{|c|c|c|c|c|c|c|c|c|}
\hline References & $\begin{array}{l}\text { Mode of } \\
\text { Exercise }\end{array}$ & Subjects & $\begin{array}{l}\text { Training } \\
\text { Status }\end{array}$ & Intensity Level & $\begin{array}{l}\text { Intervention } \\
\text { Length }\end{array}$ & $\begin{array}{l}\text { Biological } \\
\text { Tissue }\end{array}$ & $\begin{array}{c}\text { Cortisol Collection } \\
\text { Times }\end{array}$ & Cortisol Outcomes \\
\hline Smilios et al (2007) & $\mathrm{RT}$ & $\begin{array}{l}\mathrm{n}=17 \\
\widehat{\partial}=(9 \mathrm{YM}) \\
(8 \mathrm{EM})\end{array}$ & $\begin{array}{l}\text { Health } \\
\text { Physically } \\
\text { Fit }\end{array}$ & $\begin{array}{l}3 \text { x } 15 \text { reps @ } 60 \% \\
\text { of } \\
\text { 1-RM ( } 90 \text { s rest); } 6 \\
\text { exercises }\end{array}$ & Acute & Serum & Pre, IPE, \& $15 \min$ PE & $\begin{array}{l}\mathrm{C} \text { sig. } \uparrow \mathrm{IPE} \text { in both } \\
\mathrm{YM} / \mathrm{EM} ;\end{array}$ \\
\hline Raastad et al (2000) & RT & $\begin{array}{l}\mathrm{n}=9 \\
\hat{o}=9\end{array}$ & $\begin{array}{l}\text { Trained - } \\
\text { strength } \\
\text { Athletes }\end{array}$ & $\begin{array}{l}\text { High - } 100 \% \text { of } 3 \\
\text { reps max for squats } \\
\text { and } 100 \% \text { of } 6 \text { reps } \\
\text { max for leg extension; } \\
\text { Moderate }-70 \% \text { of } \\
\text { High (rest } 4-6 \text { mins) }\end{array}$ & Acute & Serum & $\begin{array}{l}\text { Pre, } 30 \text { mins into exercise, } \\
\text { 15-, 30-, 45-, \& 60-mins PE, } \\
\& 3,7,11,22,33 \text { hrs. PE. }\end{array}$ & $\begin{array}{l}\mathrm{C} \uparrow \text { greater during High vs. } \\
\text { Moderate; But } \downarrow \text { in both } \\
\text { protocols throughout } \\
\text { exercise and PE. }\end{array}$ \\
\hline Goto et al (2008) & RT & $\begin{array}{l}\mathrm{n}=9 \\
\hat{\sigma}=9(\mathrm{YM})\end{array}$ & $\begin{array}{l}\text { Health } \\
\text { Physically } \\
\text { Fit }\end{array}$ & $\begin{array}{l}5 \text { sets knee extension, } \\
\text { HI normal movement } \\
\text { (NM) } 80 \% \text {, LI Slow } \\
\text { Movement } 40 \% \text {, LI } \\
\text { normal NM } 40 \%\end{array}$ & Acute & Serum & Pre, 5-, 15-, \& 30-mins PE. & $\begin{array}{l}\mathrm{C} \downarrow \text { low normal across all } \\
\text { time points; high normal } \uparrow 5 \\
\text { min IPE returned to B @ } \\
30 \text { mins; low slow } \\
\text { movement Sig. } \uparrow \text { the most } 5 \\
\text { min PE \& returning to B in } \\
30 \text { mins }\end{array}$ \\
\hline Fujita et al (2007) & $\mathrm{RT}$ & $\begin{array}{l}\mathrm{n}=6 \\
\hat{\sigma}=6(\mathrm{YM})\end{array}$ & $\begin{array}{l}\text { Health } \\
\text { Physically } \\
\text { Fit }\end{array}$ & $\begin{array}{l}\text { 1-RM (BF restriction } \\
\text { @ } 20 \%) ; 1-\mathrm{RM} \text { no BF } \\
\text { restriction }\end{array}$ & Acute & Serum & $\begin{array}{l}\text { Pre, IPE, 20, 40, 60, 120, \& } \\
180 \text { mins }\end{array}$ & $\begin{array}{l}\text { No sig. changes with 1-RM } \\
\text { no BF restriction until 150 } \\
\text { min; Sig. } \uparrow @ 10 \text { min PE in } \\
\text { (BF restriction group) } \\
\text { returned to B@180 min. }\end{array}$ \\
\hline Szivak et al (2013) & $\mathrm{RT}$ & $\begin{array}{l}\mathrm{n}=18 \\
\hat{O}=9 \\
\mathrm{O}=9\end{array}$ & Trained & $\mathrm{HI}-75 \% 1-\mathrm{RM}$ & Acute & Serum & Pre, IPE, $15 \& 60$ mins & $\begin{array}{l}\mathrm{C} \uparrow \text { in both groups@ } @ \text { IPE, } \\
\text { 15,60 mins.Peaked @15 } \\
\text { mins }\end{array}$ \\
\hline
\end{tabular}


2021, Volume 4 (Issue 4): 19

\begin{tabular}{|c|c|c|c|c|c|c|c|c|}
\hline Arazi et al (2013) & RT & $\begin{array}{l}\mathrm{n}=18 \\
\hat{O}=(10 \mathrm{YM}), \\
(8 \mathrm{MM})\end{array}$ & Untrained & $\begin{array}{l}\text { Bench, squat } \\
\text { (moderate) }\end{array}$ & $3 \times 8$ weeks & Serum & Pre \& Post (8 weeks) & $\begin{array}{l}\text { Both groups saw sig. } \downarrow \text { from } \\
\text { Pre-to Post }\end{array}$ \\
\hline $\begin{array}{l}\text { McCaulley et al } \\
\text { (2008) }\end{array}$ & RT & $\begin{array}{l}\mathrm{n}=10 \\
\widehat{\partial}=10\end{array}$ & $\begin{array}{l}\text { Trained } \\
\text { (Strength/ } \\
\text { Power) }\end{array}$ & $\begin{array}{l}\text { H - } 75 \% 1-R M \\
\text { S - } 90 \% 1-R M \\
\text { P- } 0 \% 1-R M\end{array}$ & Acute & Serum & $\begin{array}{l}\text { Pre, IPE, } 60 \mathrm{~min}, 24, \& \\
48 \mathrm{Hr} .\end{array}$ & $\begin{array}{l}\mathrm{H}-\uparrow \mathrm{Sig}(12.4 \%) @ \mathrm{IPE} \\
\mathrm{S}-\downarrow \text { Sig. }(18.2 \%) @ \text { IPE } \\
\mathrm{P}-\downarrow \text { Sig. }(23 \%) @ \text { IPE }\end{array}$ \\
\hline $\begin{array}{l}\text { Häkkinen and } \\
\text { Pakarinen (1995) }\end{array}$ & RT & $\begin{array}{l}\mathrm{n}=47 \\
\hat{0}=24(\mathrm{YM}, \\
\mathrm{MM}, \mathrm{EM}) \\
+=23 \\
(\mathrm{YW}, \mathrm{MW} \\
\mathrm{EW})\end{array}$ & Untrained & 5 sets $\times 10$ reps $1-\mathrm{RM}$ & Acute & Serum & $\begin{array}{l}\text { Pre, } 4,6,7, \& \\
8 \text { hours PE }\end{array}$ & $\begin{array}{l}\text { No sig. C changes occurred } \\
\text { in any of the women’s } \\
\text { group; all men groups } \uparrow \\
\text { during exercise, with MM } \\
\text { having the only sig. } \uparrow \text {. }\end{array}$ \\
\hline Kim et al (2014) & RT & $\begin{array}{l}\mathrm{n}=13(\mathrm{YW}) \\
\mathrm{q}=13\end{array}$ & $\begin{array}{l}\text { Health } \\
\text { Physically } \\
\text { Fit }\end{array}$ & $\begin{array}{l}\text { Low intensity } 1-\mathrm{RM} \\
@ 20 \% \text { with } 2 \text { sets of } \\
15 \text { reps; } 80 \% 1-\mathrm{RM} 3 \\
\text { x } 10 ; \text { BF was } \\
\text { restricted }\end{array}$ & Acute & Serum & Pre-and IPE & $\begin{array}{l}\text { C sig. } \uparrow \text { IPE for both } \\
\text { protocols. No sig. } \\
\text { differences between } \\
\text { protocols. }\end{array}$ \\
\hline $\begin{array}{l}\text { Villanueva et al } \\
\text { (2012) }\end{array}$ & RT & $\begin{array}{l}n=6 \\
\delta=6\end{array}$ & $\begin{array}{l}\text { Health } \\
\text { Physically } \\
\text { Fit }\end{array}$ & $\begin{array}{l}\text { S- } 8 \times 3 @ 85 \% \\
\text { H- } 3 \times 10 @ 70 \%\end{array}$ & Acute & Serum & Pre, IPE, $15 \& 30$ mins & $\begin{array}{l}\text { C did not sig. change during } \\
\text { any of the exercise sessions }\end{array}$ \\
\hline $\begin{array}{l}\text { Fatouros et al } \\
(2009)\end{array}$ & RT & $\begin{array}{l}\mathrm{n}=40 \\
\text { (inactive) } \\
\hat{\jmath}=40\end{array}$ & Sedentary & $\begin{array}{l}\text { B, low, moderate, } \\
\text { high intensity }\end{array}$ & Acute & Serum & $\begin{array}{l}\text { Pre, IPE, 12, 24, 48, \& } 72 \\
\text { hours PE }\end{array}$ & $\begin{array}{l}\text { C peaked IPE in all } \\
\text { conditions and remained } \\
\text { elevated } 12 \text { hours except B } \\
\text { which decreased }\end{array}$ \\
\hline $\begin{array}{l}\text { Häkkinen et al } \\
\text { (1998) }\end{array}$ & RT & $\begin{array}{l}\mathrm{n}=20 \\
\mathrm{o}=(10 \mathrm{YM}), \\
(10 \mathrm{EM})\end{array}$ & $\begin{array}{l}\text { Health } \\
\text { Physically } \\
\text { Fit }\end{array}$ & $\begin{array}{l}4 \times 1 \times 10=40 \\
\text { maximal isometric } \\
\text { contractions }\end{array}$ & Acute & Serum & $\begin{array}{l}\text { Pre - lower and upper } \\
\text { extremity; Post - lower, } \\
\text { and upper extremity }\end{array}$ & $\begin{array}{l}\text { No Sig. Changes were } \\
\text { observed in YM or EM }\end{array}$ \\
\hline Kruger et al (2011) & RT & $\begin{array}{l}\mathrm{n}=15 \\
\mathrm{O}=15\end{array}$ & $\begin{array}{l}\text { Health } \\
\text { Physically } \\
\text { Fit }\end{array}$ & $\begin{array}{l}\mathrm{HI}-75 \% \text { of the } 1 \mathrm{RM} \\
(2 \text { min breaks); MI - } \\
60 \% \\
1-\mathrm{RM} \text { (total } 90 \text { mins' } \\
\text { protocol) }\end{array}$ & Acute & Serum & $\begin{array}{l}\text { Pre, IPE, } 3 \& 24 \\
\text { hours PE }\end{array}$ & $\begin{array}{l}\mathrm{C} \text { sig. } \uparrow 3 \mathrm{hrs.} \text { PE in HI, but } \\
\text { no sig. changes in MI. }\end{array}$ \\
\hline
\end{tabular}

Abbreviations: $\mathrm{A}=$ Aerobic; $\mathrm{B}=$ Baseline; $\mathrm{BF}=$ Blood Flow; $\mathrm{CE}=$ Cycle Ergometer; $\mathrm{C}=$ Cortisol; $\mathrm{E}=$ Endurance; $\mathrm{EM}=$ Elderly Men; EW = Elderly Women; HIIE = High Intensity Interval Exercise; MM = Middle-age Men; MW = Middle-age Women; PEE = Prolonged Endurance Exercise; H = Hypertrophy; IPE = immediately Post-Exercise; P = Power; PE = Post Exercise; R = Running; RT = Resistance Training; S = Strength; SSE = Steady State Exercise; WL = Workload; YM = Young Men; YW = Young Women; $\downarrow=$ Decreased; $\uparrow=$ Increased; $\hat{\delta}=$ Male; $=$ Female. 
Thus, indicating that exercise intensity is still one of the main factors influencing greater cortisol production. Magalhaes et al. ${ }^{41}$ compared the differences between moderate continuous training to RT and high-intensity interval training (HIIT) and found no significant difference in cortisol levels between both groups from the start of the intervention to the end. The lack of changes could be due to the exercise adaptations the patients may have gained over the course of the exercise intervention. As seen in previous studies, training adaptations seem to reduce the sensitivity of cortisol in individuals. ${ }^{25,29}$ Similar results were observed by Ambrozy et al. ${ }^{42}$ who examined a decrease in the cortisol levels of men between the ages of 35-40 years old. The men performed eight weeks of HIIT and RT and demonstrated a more balanced testosterone/cortisol ratio. An increase in testosterone indicated which training adaptation occurred and resulted in a blunting or loss in cortisol sensitivity. ${ }^{25,42}$

\section{Conclusion}

Exercise intensities significantly affect cortisol production differently in aerobic and RT. Cortisol has a higher level of expression in aerobic exercises with extended time periods of increased sympathetic activation or RT that utilizes high intervals for prolonged periods. Overall aerobic exercise stimulates greater production of cortisol when compared to RT. Table 2 shows that exercise time periods of 60 minutes or greater at $65 \%$ to $90 \%$ (moderate-to-high) intensity will elicit the greatest increases in cortisol. Also, multiple repeated days of high-intensity endurance exercise will elevate cortisol concentrations 2.2 to 2.6-fold greater post-exercise in cross country skiers ${ }^{18}$. Cortisol concentrations were studied in short sprint protocols $(4 \times 250 \mathrm{M})$ with three-minute rest in-between, with no significant increase in cortisol production ${ }^{47}$. RT with longer rest periods appears to attenuate cortisol production, but increased cortisol activation is elevated with shorter rest periods, as shown in Table 2. There exist marginal differences in cortisol production with aerobic exercise between men and women ${ }^{28}$. However, the research is limited to only a single study when compared with 12 studies focused on men. Farrell et al. ${ }^{28}$ introduced an acute bout of incremental (65\% and 80\%) aerobic exercise in three men and three women who were healthy and fit. Regardless of gender, cortisol increased the same in both males and females post-exercise.

Regarding RT, there appear to be varying results depending on the training status of both males and females 9,14. Age is also a significant factor; as individuals age, the rate of cortisol production in response to different exercise intensities and modalities varies. When comparing studies with both older women and men, the cortisol responses to exercise in men are increased but are unchanged in women ${ }^{14}$. The reason for this could be that the men exercised at higher intensities when compared to the women. In addition, the amount of time that was spent exercising was significantly less when compared with previous studies that observed increases in cortisol production ${ }^{9}$. Also, cortisol production is significantly higher with aerobic exercise when compared to RT. Studies with younger men and women presented more uniform responses with significant increases in cortisol production being similar in both genders ${ }^{3}$. The importance that elevated cortisol concentrations have in conjunction with decreases in lean muscle mass is more significant to elderly health and functional abilities than when compared to normal healthy younger individuals. Currently, the vast majority of studies involving the effect of exercise intensities on cortisol production are primarily in young to middle-aged men 3,9,13,30. Currently, limited studies exist that compare older men to younger men. Research involving cortisol production in women is substantially understudied when compared to men 14,19,44. Research in women is significantly needed, especially when comparing pre-menopausal to post-menopausal, sedentary to active, and trained too untrained. In conclusion, a literature review established that varying exercise intensity levels undeniably affect cortisol production during exercise. Nonetheless, the length of time spent exercising at higher-intensity levels appears to be the main contributing factor in increasing cortisol production. This may be attributed to greater sympathetic activation stimulated by the release of ACTH or by altered circulating glucose levels during exercise. 
Table 3. Production of Cortisol in Response to Combined RT and Aerobic Exercise Intensities

\begin{tabular}{|c|c|c|c|c|c|c|c|c|}
\hline References & $\begin{array}{l}\text { Mode of } \\
\text { Exercise }\end{array}$ & Subjects & $\begin{array}{l}\text { Training } \\
\text { Status }\end{array}$ & Intensity Level & $\begin{array}{l}\text { Intervention } \\
\text { Length }\end{array}$ & $\begin{array}{l}\text { Biological } \\
\text { Tissue }\end{array}$ & $\begin{array}{l}\text { Cortisol } \\
\text { Collection } \\
\text { Times }\end{array}$ & Cortisol Outcomes \\
\hline WJ Kraemer (1995) & RT/A & $\begin{array}{l}\mathrm{n}=35 \\
\hat{o}=35\end{array}$ & $\begin{array}{l}\text { Health } \\
\text { Physically } \\
\text { Fit }\end{array}$ & $\begin{array}{l}\mathrm{HI} / \mathrm{SE}, \mathrm{HI} / \mathrm{S}, \mathrm{HI} / \mathrm{E} \\
\text { Upper Body HI/SE, } \\
\text { Control }\end{array}$ & 2 x 12 weeks; & Serum & Pre, $4,8,12$ weeks & $\begin{array}{l}\mathrm{HI} / \mathrm{SE}-\uparrow \text { pre- } 12 \text { weeks; } \\
\mathrm{HI} / \mathrm{S}-\text { initially } \uparrow \text { then } \downarrow \text { by } \\
\text { week } 12 \text {; HI } / \mathrm{E}-\uparrow \text { pre- } 12 \\
\text { weeks; Upper Body HI/SE - } \\
\text { initially } \uparrow \text { then } \downarrow \text { by week } 12\end{array}$ \\
\hline Vale (2009) & $\mathrm{RT} / \mathrm{A}$ & $\begin{array}{l}\mathrm{n}=35(\mathrm{EW}) \\
q=12 \mathrm{RT} \\
13 \mathrm{E}, 10 \text { controls }\end{array}$ & Healthy & $\begin{array}{l}\text { RT - 1-RM (75-85\%), } \\
\text { E - Aquatic, Control - } \\
\text { nothing }\end{array}$ & $\begin{array}{l}3 \times 12 \text { weeks, } 2 \\
\text { sets, } 8-10 \text { reps }\end{array}$ & Serum & Pre and Post & $\begin{array}{l}\text { No sig. changes in C levels } \\
\text { from Pre to Post }\end{array}$ \\
\hline Sherk (2010) & $\mathrm{RT} / \mathrm{A}$ & $\begin{array}{l}\mathrm{n}=10 \\
\hat{\sigma}=10\end{array}$ & Trained & $\begin{array}{l}\text { Rock climbers } \\
\text { climbed for } 30 \text { mins } \\
\text { or until exhaustion }\end{array}$ & Acute & Serum & $\begin{array}{l}\text { Pre, IPE, } 15 \text { min } \\
\text { PE }\end{array}$ & $\begin{array}{l}\mathrm{C} \text { did not sig. change during } \\
\text { the protocol }\end{array}$ \\
\hline Magalhães (2020) & $\mathrm{RT} / \mathrm{A}$ & $\begin{array}{l}\mathrm{n}=80 \\
\hat{\delta}=42 \\
\stackrel{\lambda}{+}=38\end{array}$ & Healthy & $\begin{array}{l}3 \text { phases: Phase } 1 \\
\text { identical for both } \\
\text { groups, } 40-60 \% \text { of } \\
\text { HRR. Phase } 2 \text { for } \\
\text { MCT exercised at } 40- \\
60 \% \text { of HRR \& HIIE } \\
\text { exercised at } 70-80 \% \text {. } \\
\text { Phase } 3 \text { for HIIE } \\
\text { only, exercised at } \\
90 \% \text { of HRR. }\end{array}$ & $\begin{array}{l}12 \text { months, } \\
3 \mathrm{x} \text { a week }\end{array}$ & Serum & Pre and Post & $\begin{array}{l}\text { No sig. changes in C levels } \\
\text { from Pre to Post }\end{array}$ \\
\hline \multicolumn{9}{|c|}{ 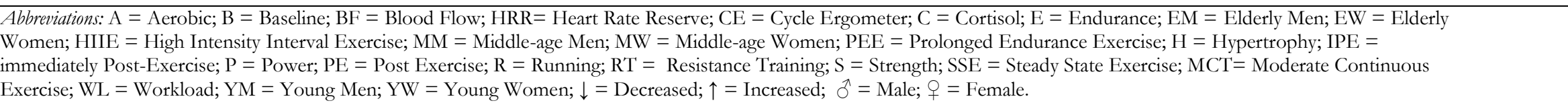 } \\
\hline
\end{tabular}




\section{References}

1. Hill EE, Zack E, Battaglini C, Viru M, Viru A, Hackney AC. Exercise and circulating cortisol levels: the intensity threshold effect. J Endocrinol Invest. 2008;31(7):587-591.

2. Wittert GA, Livesey JH, Espiner EA, Donald RA. Adaptation of the hypothalamopituitary adrenal axis to chronic exercise stress in humans. Med Sci Sports Exerc. 1996;28(8):1015-1019.

3. Wahl P, Zinner C, Achtzehn S, Bloch W, Mester J. Effect of high- and low-intensity exercise and metabolic acidosis on levels of GH, IGF-I, IGFBP-3 and cortisol. Growth Hormone \& IGF Research. 2010;20(5):380385. doi:10.1016/j.ghir.2010.08.001

4. Hackney AC. Stress and the neuroendocrine system: the role of exercise as a stressor and modifier of stress. Expert Rev Endocrinol Metab. 2006;1(6):783-792. doi:10.1586/17446651.1.6.783

5. Kraemer WJ, Ratamess NA. Hormonal responses and adaptations to resistance exercise and training. Sports Med. 2005;35(4):339-361.

6. Thau L, Gandhi J, Sharma S. Physiology, Cortisol. In: StatPearls. StatPearls Publishing; 2021. Accessed October 13, 2021. http://www.ncbi.nlm.nih.gov/books/NBK538239/

7. Di Blasio A, Izzicupo P, Tacconi L, et al. Acute and delayed effects of high-intensity interval resistance training organization on cortisol and testosterone production. J Sports Med Phys Fitness. Published online November 6, 2014.

8. Rämson R, Jürimäe J, Jürimäe T, Mäestu J. Behavior of testosterone and cortisol during an intensitycontrolled high-volume training period measured by a training task-specific test in men rowers. J Strength Cond Res. 2009;23(2):645-651. doi:10.1519/JSC.0b013e318196b801

9. Szivak TK, Hooper DR, Dunn-Lewis C, et al. Adrenal cortical responses to high-intensity, short rest, resistance exercise in men and women. J Strength Cond Res. 2013;27(3):748-760. doi:10.1519/JSC.0b013e318259e009

10. McCaulley GO, McBride JM, Cormie P, et al. Acute hormonal and neuromuscular responses to hypertrophy, strength and power type resistance exercise. Eur J Appl Physiol. 2008;105(5):695-704. doi:10.1007/s00421008-0951-z

11. Fujita S, Abe T, Drummond MJ, et al. Blood flow restriction during low-intensity resistance exercise increases S6K1 phosphorylation and muscle protein synthesis. Journal of Applied Physiology. 2007;103(3):903-910. doi:10.1152/japplphysiol.00195.2007

12. Kraemer WJ, Patton JF, Gordon SE, et al. Compatibility of high-intensity strength and endurance training on hormonal and skeletal muscle adaptations. Journal of Applied Physiology. 1995;78(3):976-989.

13. Arazi H, Damirchi A, Asadi A. Age-related hormonal adaptations, muscle circumference and strength development with 8weeks moderate intensity resistance training. Annales d'Endocrinologie. 2013;74(1):3035. doi:10.1016/j.ando.2012.11.004

14. Häkkinen K, Pakarinen A. Acute hormonal responses to heavy resistance exercise in men and women at different ages. Int J Sports Med. 1995;16(8):507-513. doi:10.1055/s-2007-973045

15. Häkkinen K, Pakarinen A, Newton RU, Kraemer WJ. Acute hormone responses to heavy resistance lower and upper extremity exercise in young versus old men. Eur J Appl Physiol Occup Physiol. 1998;77(4):312319. doi:10.1007/s004210050339

16. Hiruntrakul A, Nanagara R, Emasithi A, Borer KT. Effect of endurance exercise on resting testosterone levels in sedentary subjects. Cent Eur J Public Health. 2010;18(3):169-172.

17. Hew-Butler T, Noakes TD, Soldin SJ, Verbalis JG. Acute changes in endocrine and fluid balance markers during high-intensity, steady-state, and prolonged endurance running: unexpected increases in oxytocin and brain natriuretic peptide during exercise. Eur J Endocrinol. 2008;159(6):729-737. doi:10.1530/EJE-08-0064

18. Viru M, Hackney AC, Janson T, Karelson K, Viru A. Characterization of the cortisol response to incremental exercise in physically active young men. Acta Physiol Hung. 2008;95(2):219-227. doi:10.1556/APhysiol.95.2008.2.6

19. Vale RG de S, de Oliveira RD, Pernambuco CS, de Meneses YP da SF, Novaes J da S, de Andrade A de FD. Effects of muscle strength and aerobic training on basal serum levels of IGF-1 and cortisol in elderly women. Archives of Gerontology and Geriatrics. 2009;49(3):343-347. doi:10.1016/j.archger.2008.11.011 
20. Deuster PA, Chrousos GP, Luger A, et al. Hormonal and metabolic responses of untrained, moderately trained, and highly trained men to three exercise intensities. Metabolism. 1989;38(2):141-148. doi:10.1016/0026-0495(89)90253-9

21. Maresh C, Whittlesey M, Armstrong L, et al. Effect of Hydration State on Testosterone and Cortisol Responses to Training-Intensity Exercise in Collegiate Runners. International Journal of Sports Medicine. 2006;27(10):765-770. doi:10.1055/s-2005-872932

22. Peake J, Wilson G, Mackinnon L, Coombes JS. Carbohydrate supplementation and alterations in neutrophils, and plasma cortisol and myoglobin concentration after intense exercise. Eur J Appl Physiol. 2004;93(5-6):672678. doi:10.1007/s00421-004-1248-5

23. Martínez-Díaz IC, Carrasco L. Neurophysiological Stress Response and Mood Changes Induced by HighIntensity Interval Training: A Pilot Study. Int J Environ Res Public Health. 2021;18(14):7320. doi:10.3390/ijerph18147320

24. Rojas Vega S, Strüder HK, Vera Wahrmann B, Schmidt A, Bloch W, Hollmann W. Acute BDNF and cortisol response to low intensity exercise and following ramp incremental exercise to exhaustion in humans. Brain Research. 2006;1121(1):59-65. doi:10.1016/j.brainres.2006.08.105

25. Jörres M, Gunga H-C, Steinach M. Physiological Changes, Activity, and Stress During a 100-km-24-h Walking-March. Front Physiol. 2021;12:640710. doi:10.3389/fphys.2021.640710

26. Tremblay MS, Copeland JL, Helder WV. Influence of exercise duration on post-exercise steroid hormone responses in trained males. Eur J Appl Physiol. 2005;94(5-6):505-513. doi:10.1007/s00421-005-1380-x

27. Kuoppasalmi K, Näveri H, Härkönen M, Adlercreutz H. Plasma cortisol, androstenedione, testosterone and luteinizing hormone in running exercise of different intensities. Scand J Clin Lab Invest. 1980;40(5):403-409. doi:10.3109/00365518009101862

28. Farrell PA, Garthwaite TL, Gustafson AB. Plasma adrenocorticotropin and cortisol responses to submaximal and exhaustive exercise. J Appl Physiol Respir Environ Exerc Physiol. 1983;55(5):1441-1444.

29. Stenqvist TB, Torstveit MK, Faber J, Melin AK. Impact of a 4-Week Intensified Endurance Training Intervention on Markers of Relative Energy Deficiency in Sport (RED-S) and Performance Among WellTrained Male Cyclists. Front Endocrinol (Lausanne). 2020;11:512365. doi:10.3389/fendo.2020.512365

30. Witard OC, Turner JE, Jackman SR, et al. High-intensity training reduces CD8+ T-cell redistribution in response to exercise. Med Sci Sports Exerc. 2012;44(9):1689-1697. doi:10.1249/MSS.0b013e318257d2db

31. Vuorimaa T, Ahotupa M, Häkkinen K, Vasankari T. Different hormonal response to continuous and intermittent exercise in middle-distance and marathon runners. Scandinavian Journal of Medicine \& Science in Sports. 2008;18(5):565-572. doi:10.1111/j.1600-0838.2007.00733.x

32. Tabata I, Atomi Y, Miyashita M. Blood glucose concentration dependent ACTH and cortisol responses to prolonged exercise. Clinical Physiology. 1984;4(4):299-307. doi:10.1111/j.1475-097X.1984.tb00805.x

33. Caplin A, Chen FS, Beauchamp MR, Puterman E. The effects of exercise intensity on the cortisol response to a subsequent acute psychosocial stressor. Psychoneuroendocrinology. 2021;131:105336. doi:10.1016/j.psyneuen.2021.105336

34. Arvidson E, Dahlman AS, Börjesson M, Gullstrand L, Jonsdottir IH. The effects of exercise training on hypothalamic-pituitary-adrenal axis reactivity and autonomic response to acute stress-a randomized controlled study. Trials. 2020;21(1):888. doi:10.1186/s13063-020-04803-3

35. Raastad T, Bjøro T, Hallén J. Hormonal responses to high- and moderate-intensity strength exercise. Eur J Appl Physiol. 2000;82(1-2):121-128. doi:10.1007/s004210050661

36. Villanueva MG, Villanueva MG, Lane CJ, Schroeder ET. Influence of rest interval length on acute testosterone and cortisol responses to volume-load-equated total body hypertrophic and strength protocols. J Strength Cond Res. 2012;26(10):2755-2764. doi:10.1519/JSC.0b013e3182651fbe

37. Gepfert M, Trybulski R, Stastny P, Wilk M. Fast Eccentric Movement Tempo Elicits Higher Physiological Responses than Medium Eccentric Tempo in Ice-Hockey Players. Int J Environ Res Public Health. 2021;18(14):7694. doi:10.3390/ijerph18147694

38. Krüger K, Agnischock S, Lechtermann A, et al. Intensive resistance exercise induces lymphocyte apoptosis via cortisol and glucocorticoid receptor-dependent pathways. Journal of Applied Physiology. 2011;110(5):1226-1232. doi:10.1152/japplphysiol.01295.2010

39. Goto K, Takahashi K, Yamamoto M, Takamatsu K. Hormone and Recovery Responses to Resistance Exercise with Slow Movement. The Journal of Physiological Sciences. 2008;58(1):7-14. doi:10.2170/physiolsci.RP003107

40. Sherk VD, Sherk KA, Kim S, Young KC, Bemben DA. Hormone responses to a continuous bout of rock climbing in men. Eur J Appl Physiol. 2010;111(4):687-693. doi:10.1007/s00421-010-1685-2 
41. Magalhães JP, Santos DA, Correia IR, et al. Impact of combined training with different exercise intensities on inflammatory and lipid markers in type 2 diabetes: a secondary analysis from a 1 -year randomized controlled trial. Cardiovasc Diabetol. 2020;19(1):169. doi:10.1186/s12933-020-01136-y

42. Ambroży T, Rydzik Ł, Obmiński Z, et al. The Effect of High-Intensity Interval Training Periods on Morning Serum Testosterone and Cortisol Levels and Physical Fitness in Men Aged 35-40 Years. J Clin Med. 2021;10(10):2143. doi:10.3390/jcm10102143

43. Smilios I, Pilianidis T, Karamouzis M, Parlavantzas A, Tokmakidis S. Hormonal Responses after a Strength Endurance Resistance Exercise Protocol in Young and Elderly Males. International Journal of Sports Medicine. 2007;28(5):401-406. doi:10.1055/s-2006-924366

44. Kim E, Gregg LD, Kim Ld, Sherk VD, Bemben MG, Bemben DA. Hormone Responses to an Acute Bout of Low Intensity Blood Flow Restricted Resistance Exercise in College-Aged Females. J Sports Sci Med. 2014;13(1):91-96.

45. Bucciarelli V, Bianco F, Mucedola F, et al. Effect of Adherence to Physical Exercise on Cardiometabolic Profile in Postmenopausal Women. Int J Environ Res Public Health. 2021;18(2):E656. doi:10.3390/ijerph18020656

46. Bessinger RC, McMurray RG, Hackney AC. Substrate utilization and hormonal responses to moderate intensity exercise during pregnancy and after delivery. American Journal of Obstetrics and Gynecology. 2002;186(4):757-764. doi:10.1067/mob.2002.122093

47. Meckel Y, Eliakim A, Seraev M, et al. The effect of a brief sprint interval exercise on growth factors and inflammatory mediators. J Strength Cond Res. 2009;23(1):225-230. doi:10.1519/JSC.0b013e3181876a9a

48. Martínez-Díaz IC, Carrasco L. Neurophysiological Stress Response and Mood Changes Induced by HighIntensity Interval Training: A Pilot Study. Int J Environ Res Public Health. 2021;18(14):7320. doi:10.3390/ijerph18147320

49. Gepfert M, Trybulski R, Stastny P, Wilk M. Fast Eccentric Movement Tempo Elicits Higher Physiological Responses than Medium Eccentric Tempo in Ice-Hockey Players. Int J Environ Res Public Health. 2021;18(14):7694. doi:10.3390/ijerph18147694 